\title{
Egypt, The Coronavirus Test \& International Transformations
}

\author{
By: AbdelMoeti Abuzeid \\ Editor- in -Shief \\ Head of Foreign Media Sector
}

The Covid-19 pandemic, widely known as "Coronavirus", is a severe test for all nations and peoples, a test for health systems, administrative and logistical capabilities, and the extent of trust between the peoples and their leaders in the battle for awareness and comprehensive response to the repercussions. This pandemic has also been and continues to be a real test for the economic systems of the world' s countries and their ability to manage the crisis, and to adapt to its sudden profound negative effects.

In all these tests, Egypt has achieved remarkable success and shown great resilience. Egypt's health system has proven its ability to act in an organized manner in the face of the pandemic. The country has not experienced shortage of hospital beds, medical staff or pharmaceutical supplies. The best methods of diagnostic testing and screening, isolation precautions and treatment protocols have been followed, making Egypt's infection and death rates among the lowest in the world in terms of mortality or infection per million population.

In the battle for awareness, the leadership has won a great deal of credibility in the people's minds and hearts and relevant authorities have shown strong commitment to the guidelines, both regarding transmissive and protective measures against the outbreak of the pandemic and guarantees for the continuation of businesses and production for the duration of the covid-19 pandemic.

At the economic level, Egypt has been at the forefront of the region and indeed the world - in reducing the negative effects of the pandemic on the Egyptian economy. Economic growth continued -- although, of course, lower than previous years and pre-pandemic forecasts. The testimonies of international institutions confirmed this success. "Standard \& Poor's" credit rating firm has established the degree of sovereign rating of the Egyptian economy at the level of B/B in the long-term and shortterm while maintaining a stable outlook, Fitch affirms Egypt at ' $\mathrm{B}+$ ' and 


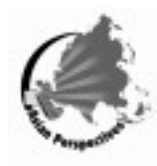

ratings agency Moody's has kept Egypt's credit rating at B2 with a stable outlook, all at the same pre-pandemic rating.

All this has been achieved thanks to Egypt's completion of the economic reform program prior to the outbreak of the coronavirus pandemic, as well as successive economic decisions that have dealt wisely, without panic or encumbrances, with the economic consequences of the pandemic.

At the social level, numerous decisions have been promulgated to reflect the State's solidarity with the most vulnerable and needy groups, most affected by the pandemic, such as irregular and precarious workers in sectors such as tourism. Concessions were also grated to small enterprises and businesses which have been negatively affected during this time.

At the same time, in addition to confronting these effects, the international political and economic system has undergone significant changes as a result of the pandemic, as well as some political changes in both the US leadership and the challenges in the Middle East and the Mediterranean regions. Egypt is dealing flexibly and decisively with these challenges, hence the importance of Egyptian-Asian relations, whether with the continent's giants such as China, India, Japan and Russia, or Asian economic groupings, in providing a wider margin of Egyptian movement within different regions of the world.

In this spirit, the current edition of "Asian Perspectives", is full of many reports and studies dealing with this new international reality. The issue's feature focuses on "The Continent of Asia \& The Coronavirus Pandemic" in all respects and regions of Asia. The current edition also includes reports on economic, political and cultural issues in many Asian countries as well as usual assorted departments such as seminars, conferences, Asian library and others.

Noteworthy, "Asian Perspectives" got an ISBN as an academic scientific publication issued in both paper and electronic versions, accredited by the Academy of Scientific Research in Egypt, which further attests to the scientific value of this periodical and the distinguished standing of its contributing writers. 